\title{
Evaluation and management of adult acquired buried penis
}

\author{
Tammy S. Ho, Joel Gelman \\ Department of Urology, University of California Irvine, Irvine, California, USA \\ Contributions: (I) Conception and design: All authors; (II) Administrative support: None; (III) Provision of study materials or patients: J Gelman; (IV) \\ Collection and assembly of data: TS Ho; (V) Data analysis and interpretation: All authors; (VI) Manuscript writing: All authors; (VII) Final approval \\ of manuscript: All authors. \\ Correspondence to: Joel Gelman, MD. Department of Urology, University of California Irvine, Irvine, 101 The City Drive, Building 29, Pavilion 3, \\ Orange, California 92868, USA. Email: jgelman@uci.edu.
}

\begin{abstract}
Adult acquired buried penis represents the clinical manifestation of a wide spectrum of pathology due to a variety of etiologies. It can be related to obesity, a laxity in connective tissue, lichen sclerosis (LS), complications from penile/scrotal enlargement surgery, scrotal lymphedema, or hidradenitis suppurativa (HS). Buried penis can be associated with poor cosmesis and hygiene, voiding dysfunction, and sexual dysfunction. Evaluation and management of buried penis largely depends on etiology and degree of affected tissue. It is an increasingly common problem seen by reconstructive urologists and here we present several frequently seen scenarios of buried penis and management options.
\end{abstract}

Keywords: Balanitis xerotica obliterans (BXO); buried penis; lichen sclerosis (LS); massive localized lymphedema (MLL)

Submitted Mar 20, 2018. Accepted for publication May 07, 2018.

doi: $10.21037 /$ tau.2018.05.06

View this article at: http://dx.doi.org/10.21037/tau.2018.05.06

\section{Introduction}

A buried penis refers to a normal sized phallus encompassed by either skin, subcutaneous tissue, and/or fat in the prepubic area. Due to reduced visible and functional length of phallus, this condition is often associated with patient dissatisfaction related to cosmetic, hygienic, voiding, and sexual dysfunction. Subsequent phimosis can lead to spraying, dribbling, urinary soilage, and skin breakdown. Often times, the meatus is not visible and can mask undiagnosed urethral disease related to lichen sclerosis (LS) also known as balanitis xerotica obliterans (BXO). Additionally, a buried penis often limits penetrative sexual intercourse. In some series, clinical depression has been noted in a majority of patients preoperatively (1). While weight loss may improve some symptoms for those who have a buried penis related to obesity, many men have significant remaining prepubic fat deposition along with ptosis of the prepubic fat pad, or escutcheon, that would benefit from surgical repair (2). Other causes of buried penis include penile skin deficiency associated with circumcision or trauma, Fournier's gangrene, or complications of penile enlargement surgery. Additionally, severe scrotal lymphedema and genital hidradenitis often result in buried penis with a severe deficiency of normal scrotal skin to use for reconstruction. Overall, acquired buried penis in adults has become an increasingly common problem seen by reconstructive urologists.

\section{Evaluation}

The basic evaluation for a buried penis begins with a comprehensive history and physical examination. The history should reveal how long the phallus has been buried and if it was associated with any weight gain or loss. The penile and scrotal skin should be assessed for changes suggestive of LS (skin whitening, glans fusion, etc.) or hidradenitis (draining sinuses, abscesses, etc.). Associated voiding symptoms such as dysuria, straining to urinate, weak stream, dribbling, urinary tract infections, or sitting to urinate should be elucidated. Sexual function should be evaluated including erectile dysfunction, pain with erection, 


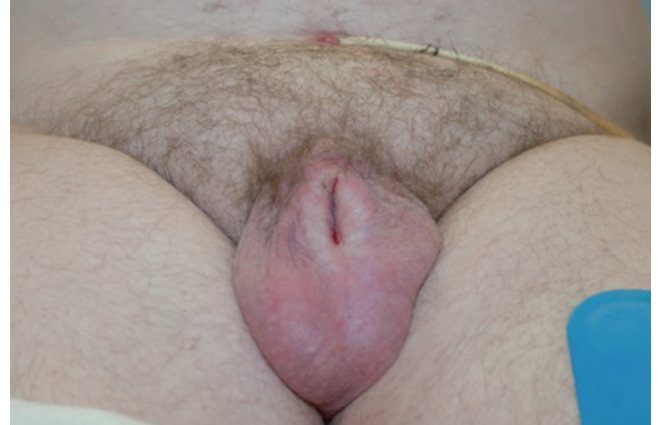

Figure 1 Due to inflammation and lichen sclerosis, a phimotic band often forms, pushing the phallus proximally and burying it.

or inability to penetrate due to buried phallus. The degree of bother and the patients' primary complaint should be documented. Is the patient concerned about cosmetic or functional outcomes? Is the goal to void standing up or to resume sexual activity? Additional history should focus on relevant comorbidities such as obesity, pulmonary issues often associated with morbid obesity, diabetes, hypertension, hidradenitis, and previous urologic history. Prior surgical history such as lymph node dissection, circumcision, penile enlargement surgery, or genital enlargement injections should be ascertained. Other causes of voiding dysfunction should be addressed such as history of benign prostatic hyperplasia or urethral stricture.

Physical examination should include general assessment of the patient's body habitus and functional status. An abdominal and suprapubic exam should be performed with notation of any overlying pannus while the patient is supine and standing. Often, patients have an overhanging suprapubic fat pad that obscures and buries the penis known as the "escutcheon". Genitourinary examination should be performed with special attention to the patient's circumcision status (circumcised or uncircumcised), meatus (presence of meatal stenosis), and penile, scrotal, and suprapubic skin quality (skin thickening, lymphedema, mobility, white discoloration concerning for LS). In particular, the skin surrounding the phallus should be examined to evaluate whether there are loose attachments, an abundance of prepubic fat pad, or a phimotic ring of scar tissue. The clinician should compress the skin and fat surrounding the buried penis in an attempt to "deliver" the penis for examination of the glans and to assess the amount of normal penile skin available. In some cases, there is an overabundance of escutcheon or a phimotic band (Figure 1) which prevents evaluation of the penile shaft skin. In those cases, the patient should be counseled about the possible need for flap or graft coverage in case there is a paucity of viable skin during reconstruction. When there is scrotal enlargement suggestive of scrotal lymphedema, the lateral scrotal and perineal skin needs to be evaluated to determine if there is sufficient supple skin for flap coverage or primary closure after excision of any effected skin and subcutaneous tissues. Moreover, where there is scrotal enlargement, the presence of any draining sinuses, abnormality of the medial thighs, or induration of the peri-anal area or armpit abnormality suggests a diagnosis of hidradenitis.

In cases where there is white skin discoloration or obliteration/fusion of the glans margin suggestive of LS, careful attention must be paid to the patient's voiding symptoms. It can often be difficult to separate voiding symptoms related to a buried penis from symptoms of urethral stricture, especially when the phallus is buried to the extent that the underlying phallus cannot be examined. These patients should be carefully counseled about the risk of concomitant urethral stricture disease. In patients with a suspicion of urethral stricture based on symptoms or a suggestion of meatal narrowing, the urethral meatus, fossa navicularis, and distal penile urethra can be calibrated with Bougie-a-boules. Cystoscopy should be performed if there is any suspicion of stricture proximal to the most distal penile urethra. If there is any narrowing of less than 16 French with calibration (which would prevent advancement of a cystoscope in the absence of "scope dilation" or a stricture noted with cystoscopy), urethral imaging with a retrograde urethrogram and possibly a voiding cystourethrogram would be indicated. Patients with buried penis and long urethral strictures need careful operative planning in order to address both of their issues. In patients with a buried penis where the meatus cannot be seen on physical exam, it is often not possible to evaluate for urethral strictures preoperatively. Prior to their buried penis operation, informed consent should include the option of cystoscopy, retrograde urethrogram, and/or suprapubic tube placement.

\section{Management}

Management of adult buried penis varies enormously based on the quality and quantity of healthy penile, scrotal, and abdominal skin. Additionally, the etiology of the buried penis plays a large role in treatment and surgical repair. In general, repair of buried penis involves unburying the penis, resection of any diseased or excess tissue, tacking tissue down to reform the penopubic angle, and coverage 
with either local skin flaps or skin grafts. Here, we have organized a series of frequently seen adult buried penis scenarios and the commonly preferred management.

\section{Obesity: hypermobility to complete invagination of the penis}

Obesity is a common cause of acquired adult acquired buried penis. There are some similarities with buried penis seen in children, which is often related to poor skin suspension, abnormal excess fat accumulation in the pubic area, webbed penis due to penoscrotal webbing, or trapped penis due to scarring post circumcision $(3,4)$. Similarly, adult buried penis is sometimes related to a laxity of connective tissue between the dartos fascia and the penis, allowing the penis to tunnel more proximally under the prepubic skin due to "hypermobility". This is exacerbated by obesity and weight gain as the suprapubic fat pad encompasses the phallus.

These patients often present with complaints of a shorter penis, difficulty having intercourse, or urinary dribbling. On physical examination, when the skin and fat surrounding the base of the penis is compressed, the shaft of the penis is typically visible. If there is no associated inflammatory skin disease such as LS, then the patient can be counseled initially regarding weight loss, which may increase the size of the visible penis by decreasing the surrounding fat. While weight loss may not lead to resolution of buried penis, this will likely help facilitate eventual repair and offer other health benefits in a population often associated with significant comorbidities. Surgical repair of "hypermobility" of the skin can include a ventral skin incision at the base of the penis and the tacking of bilateral subdermal tissue at the penoscrotal junction to the tunica albuginea lateral to the urethra (4).

While tacking hypermobile skin is often sufficient in patients with obesity, patients with more significant morbid obesity often face additional challenges to surgical repair. As patients gain weight, there is often a preferential deposition of adipose in the suprapubic area that persists even after weight loss or bariatric surgery (2). Since the phallus remains tethered to the pubis by the suspensory ligament, the redundant suprapubic fat pad eventually completely surrounds the penis. With burial of the glans and meatus, patients often have to sit to void due to dribbling. A combination of poor hygiene and persistent moisture trapped near the penis leads to chronic bacterial or fungal colonization. Chronic colonization can lead to inflammatory skin contracture and the formation of a phimotic ring of scar. This often results in invagination of the penile shaft skin and further burial of the phallus. Over time, the penile shaft skin will often break down and there will be a paucity of healthy penile tissue during time of surgical repair. Additionally, patients may have a degree of burial due to descended escutcheon or significant overlying pannus. In more severe cases of morbid obesity, surgical repair may include a formal panniculectomy, dermatolipectomy, and the tacking of the penopubic subdermis to the rectus fascia.

If a morbidly obese patient presents with significant escutcheon that limits examination of the glans, meatus, and penile skin, we counsel the patient extensively preoperatively regarding the potential for there to be a deficiency of penile skin and possible need for local flap or graft coverage. Additionally, patients are counseled about the risk of encountering undiagnosed meatal or urethral stricture associated with chronic inflammation and LS at the time of surgery since it is difficult to discern between the voiding symptoms of a buried penis alone compared to that of a buried penis associated with a urethral stricture. Additionally, if there is significant escutcheon or overlying pannus that will need to be surgically removed, a concomitant escutcheonectomy or panniculectomy may be indicated at the time of buried penis revision. In cases that involve panniculectomy, it is our preference to include the expertise of a plastic surgeon. Many patients have concomitant comorbidities such as diabetes, hypertension, and chronic obstructive pulmonary disease (COPD) that increase their risk of perioperative complications and every attempt should be made to optimize patients medically preoperatively and insure the risk of surgery is acceptable.

The surgical approach is influenced by the specific factors identified on physical examination performed with the patient in the supine and standing positions prior to surgery that may be contributing to the buried penis. These factors include the extent the problem is related to descent of the pubic skin versus excess suprapubic fat versus a deficiency of attachments of the penile skin to Buck's fascia versus a combination of factors. The suprapubic incision and amount of skin and subcutaneous suprapubic fat removed is individualized based on the above findings. The goal is to excise and lift the excess pubic skin and reduce the pubic fat, which may or may not include abdominoplasty. When there is then apparent hypermobility of the dorsal penile skin, this can then be address with tacking of the penopubic subdermis to the rectus fascia.

Often times, the penile skin can be mobilized for dorsal coverage leaving remaining ventral skin deficiency. If the 
defect is small and the scrotal skin is normal, a ventral slit scrotal flap can be used to cover the defect with a Z-plasty (5). A vertical incision is made on the scrotum with relaxing incisions to the right and left to form a $\mathrm{Z}$. The scrotal skin flap is carefully mobilized taking care to preserve the rich blood supply of the underlying dartos fascia. A rotational flap is then advanced for penile skin coverage.

Advantages of mobilizing a local scrotal flap include avoiding the morbidity associated with taking skin grafts. Scrotal skin is often supple, is easily mobilized, has no subcutaneous fat, and often offers an acceptable cosmetic result. Due to its high vascularity and dual blood supply, scrotal flap necrosis rates are rare (6). In a retrospective review of 18 patients who underwent a scrotal skin flap to the penis, 15 patients reported satisfactory sexual outcomes and sensation (6). However, scrotal skin does have the disadvantage of being hair-bearing as well as having rugae, leading to a differing cosmetic appearance compared to normal penile skin. In some cases, skin grafts are necessary for adequate coverage and will be discussed below.

\section{Lichen sclerosis}

An acquired adult buried penis may be seen in patients who develop LS, a condition that is often associated with obesity. Often times, inflammatory changes of the distal penile skin or the circumcision suture line in circumcised patients results in development of scar tissue and formation of a cicatrix (Figure 1). This traps the penis proximally, resulting in invagination and tunneling of the penis underneath the penile skin. Urine often pools beneath this tissue, leading to chronic colonization of bacteria and further inflammatory changes. When present chronically, this can lead to denudation of the tissue. In other patients, the inflamed distal penile skin does not cover the glans but is rather permanently fused to the glans giving the false appearance of phimosis.

Patients are often seen with a buried penis related to lichen sclerosis with the complaint of "phimosis". If the patient is uncircumcised and there is adequate penile skin proximal to the prepuce, circumcision represents an effective treatment for the phimosis and the associated buried penis. Moreover, in uncircumcised men, circumcision is often considered curative of LS limited to the prepuce. However, LS should be considered a chronic condition and men with the diagnosis are always at risk for recurrence involving the remaining penile skin and/or urethra. In circumcised men, management with a repeat circumcision may further worsen the skin defect and bury the shaft more proximally. We often treat initially with a course of high potency steroid cream to decrease inflammation and soften the tissue, which may avoid the need for surgery. We prefer the use of clobetasol $0.05 \%$ applied twice daily. While data is limited in adults, a systematic review showed that treatment of LS with topical steroids in children allowed avoidance of circumcision in $31 \%$ of boys (7).

In patients with voiding symptoms or the appearance of involvement of the urethral meatus, it is important to evaluate the urethra with Bougie-a-boule calibration, cystoscopy, and when there is confirmation of a stricture, urethral imaging with a retrograde urethrogram and possibly a voiding cystourethrogram. In cases of LS where there is a buried penis associated with phimosis and the meatus is not visible to permit an evaluation of the urethra, our preference is to use a technique we developed for these specific cases. The objective is to increase the circumference of the area of phimosis at the expense of skin length without removing skin which would lead to skin deficiency. This could be accomplished by a long dorsal longitudinal slit with transverse closure. However, when there is a tight constriction, considerable dorsal penile skin length loss would be required to provide adequate resolution of the phimosis. Our preference is to make four longitudinal incisions with incisions dorsally, ventrally, and bilaterally of equal length (Figure $2 A$ ) with transverse closure so that the length loss is evenly distributed along the circumference of the penis (Figure 2B). Once the glans is delivered and visible, the urethra can be evaluated. We include consent for meatotomy or extended meatotomy so that if a very short stricture is identified, it can be definitively treated at the time of surgery. In a retrospective review of 43 patients with limited involvement of LS, the aggressive use of topical steroids with minor procedures to relieve high pressure voiding may prevent disease progression (8).

If the stricture is longer and requires more extensive surgical repair, we generally favor suprabpubic tube placement with more extensive discussion of treatment options postoperatively. Significant penile deficiency can be addressed with split thickness skin grafting (STSG) of the shaft. However, when there is stricture disease extending proximal to the distal penile urethra, a management plan for the penis must take into consideration the treatment of the stricture, especially if a staged repair is being considered. During staged repairs, it could be problematic to tubularize the urethra during the second stage and cover this repair with previously grafted skin, which has a random blood 

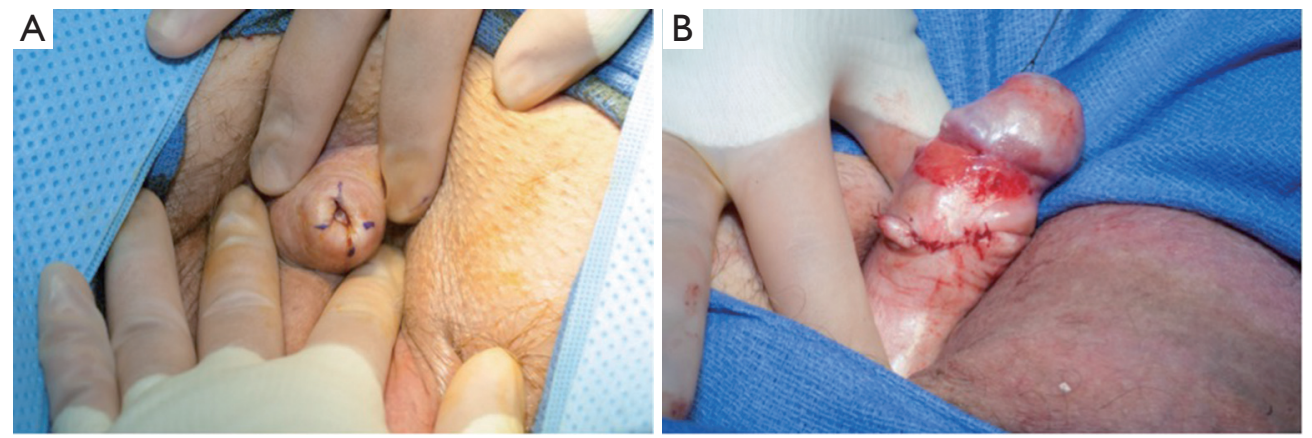

Figure 2 Technique to unbury the phallus while avoiding a large deficiency of dorsal skin. (A) Four relaxing longitudinal incisions are made dorsally, ventrally, and bilaterally rather than one long dorsal slit to decrease the dorsal skin defect; (B) the four longitudinal incisions are then closed transversely to more evenly distribute the penile skin loss along the circumference of the penis.

supply. Additionally, skin grafts typically lack mobility, which would complicate attempts at a staged urethral reconstruction. In this complicated scenario, we prefer to perform a one-stage dorsal onlay buccal mucosa graft (BMG) urethroplasty at the time of buried penis repair which has the advantage of keeping the buccal graft recipient bed away from the grafted penile skin. For example, we repaired a patient with buried penis requiring penile skin graft coverage as well as urethral stricture limited to the meatus, fossa navicularis, and penile urethra by addressing the majority of the stricture with dorsal onlay BMG and the most distal stricture with an extended meatotomy.

\section{Complications of penile enlargement surgery}

Procedures that attempt to enlarge the penis include suspensory ligament release or the placement of a variety of materials between the penile skin and tunica albuginea including subcutaneous implants, the injection of silicone, or placement of other substances into this space. Often, materials are also injected or implanted simultaneously into the scrotum. Complications can include significant deformity associated with scarring which shortens the penis and the development of subcutaneous nodules that may include fistulas and ulcers that engulf and bury the penis partially or completely.

The treatment generally involved the removal of the subcutaneous tissue masses and any hard scar tissue adherent to the tunica albuginea via a circumcising and/or peno-scrotal incision. If this skin appears viable, primary closure with either native skin or with advancement of local skin flaps is possible. However, there is a risk that the involved genital skin can then contract or become swollen, requiring subsequent surgery to excise the involved skin and use STSG for coverage. Alternatively, if it is apparent that the remaining penile skin is not viable, skin grafting can be performed at the time of the treatment of the buried penis.

As shown in Figure $3 \mathrm{~A}$, after subcutaneous silicone injections in an attempt to enlarge the penis and scrotum, the penis is noted to be buried and surrounded by a mass of subcutaneous penile and scrotal tissue. After excision of the involved tissue masses and primary closure using local skin, the penile and scrotum looked relatively normal once the surgery was completed (Figure 3B). In some cases, the penile and scrotal skin remains supple without adverse change. Thus, it is our preference to avoid grafting when the skin appears viable, but we counsel patients prior to surgery that grafting may be subsequently required if they eventual develop skin contracture or edema. In this case, the scrotal skin healed with normal appearance, but the penile skin had postoperative contracture (Figure 3C). Subsequent surgery was associated with removal of the involved skin and skin grafting. The appearance after grafting is as shown immediately after surgery (Figure $3 D$ ) and months later (Figure 3E).

\section{Scrotal lymphedema}

Buried penis can be acquired in cases of scrotal lymphedema. Rarely, this can be due to a congenital condition associated with impaired lymphatic drainage leading to scrotal enlargement. Often, scrotal lymphedema leading to buried penis is the result of postoperative sequelae from surgeries such as pelvic lymph node dissection during prostatectomy, inguinal lymphadenectomy for penile cancer or melanoma, or aortobifemoral bypass grafting. In a series of 340 inguinal 

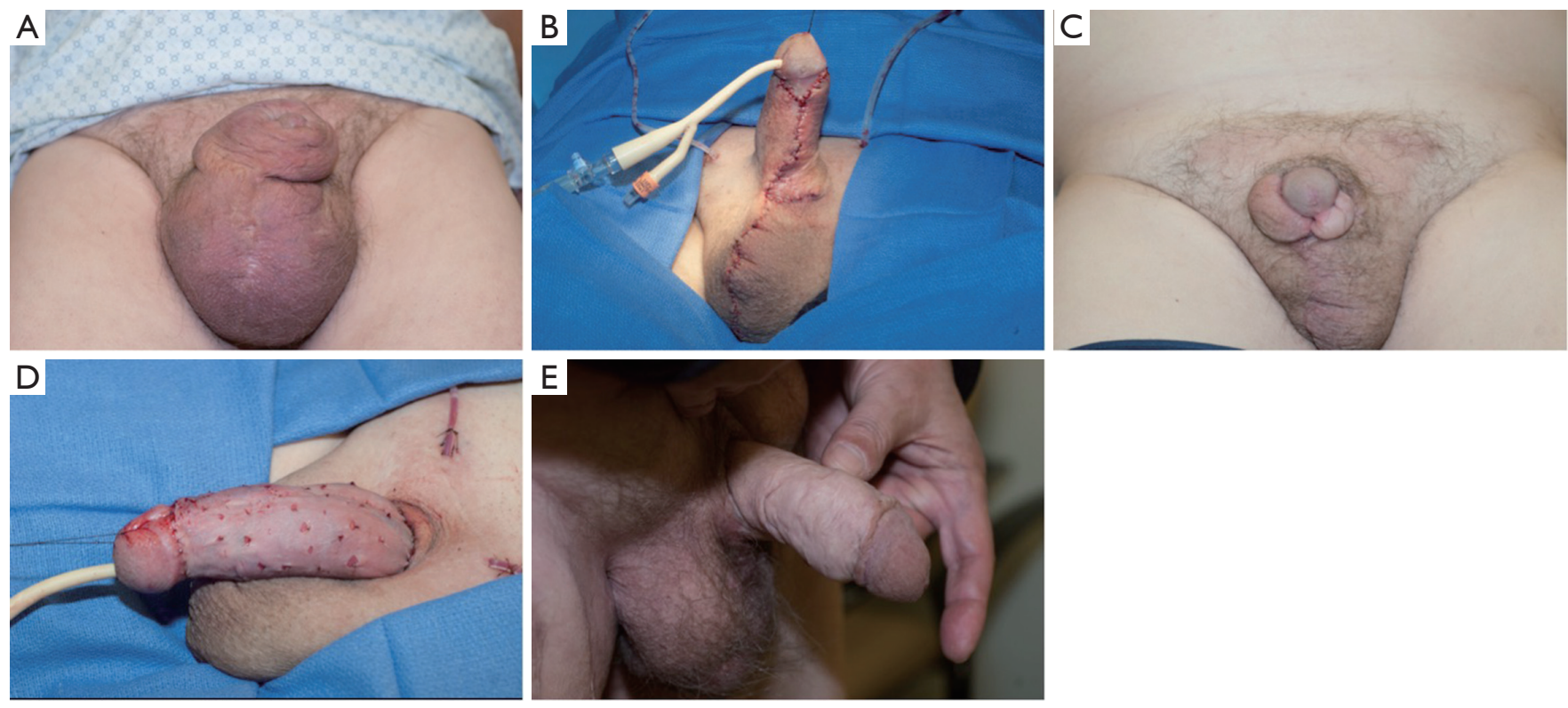

Figure 3 Buried penis repair after penile and scrotal enlargement surgery. (A) There is significant buried penis after injection of silicone into the penile and scrotal skin due to scarring and tissue masses; (B) relatively normal appearance of the penis immediately postoperatively after excision of scar tissue and abnormal penile and scrotal skin; (C) normal postoperative appearance of the scrotum associated with abnormal appearing penile skin tissue; (D) subsequent operation with excision of contracture penile skin with STSG; (E) appearance of the penis and scrotum several months postop. STSG, split thickness skin grafting.

lymphadenectomies for penile cancer, early lymphedema rates of $4.1 \%$ and scrotal edema rates of $0.9 \%$ were noted (9) with late lymphedema rates ranging from $5-9 \%(10,11)$. In patients from developing countries, scrotal lymphedema is often seen as the genital manifestation of lymphatic filariasis, also known as elephantiasis. It is most commonly transmitted by the filarial nematode Wuchereria bancroft $i$, Brugia malayi, and B. timori (12). Patients with filarial penoscrotal lymphedema are often successfully managed with surgical treatment with either STSGs or prepucial skin flaps, when available (13). In a prospective study of 11 patients undergoing surgery for giant filarial penoscrotal lymphedema in Sub-Saharan Africa, the average weight of excised scrotum was $22.4 \mathrm{~kg}$. Similar to patients with massive localized lymphedema (MLL) secondary to morbid obesity, these patients had a high complication rate with $27 \%$ of patients developing a scrotal hematoma, $18 \%$ surgical site infection, and $9 \%$ of disease recurrence in 24 months of follow-up (14).

More commonly in the United States, scrotal lymphedema severe enough to bury the penis has an unclear etiology and is often associated with morbid obesity. Scrotal masses that can engulf and bury the penis can be quite variable, sometimes growing over $60 \mathrm{~kg}$. In these cases, physical exam to rule out hernia is often of limited utility and computed tomography (CT) scan is our preferred imaging modality. In a small series of four morbidly patients with an average body mass index (BMI) of $59 \mathrm{~kg} / \mathrm{m}^{2}$ undergoing buried penis correct, patients showed improvement in urinary function and overall symptoms despite wound complications occurring in every patient (15). At some institutions, it has been suggested that preoperative complex decongestive physiotherapy (CDP), which includes skin hygiene, manual lymph drainage, compressive bandages, and therapeutic exercises, may decrease postoperative complication rates for patients with panniculus morbidus or male genital lymphedema (16). While they noted a $2-4 \%$ complication rate in their retrospective review of 51 patients undergoing surgery for male genital lymphedema, the average BMI of patients was not reported and may represent a different population compared to the MLL observed in morbidly obese patients.

Our surgical approach in patients with MLL (Figure 4A) is to make a T-shaped incision with the transverse incision located just inferior to the invagination of the scrotal skin around the penis with the vertical extension along the 

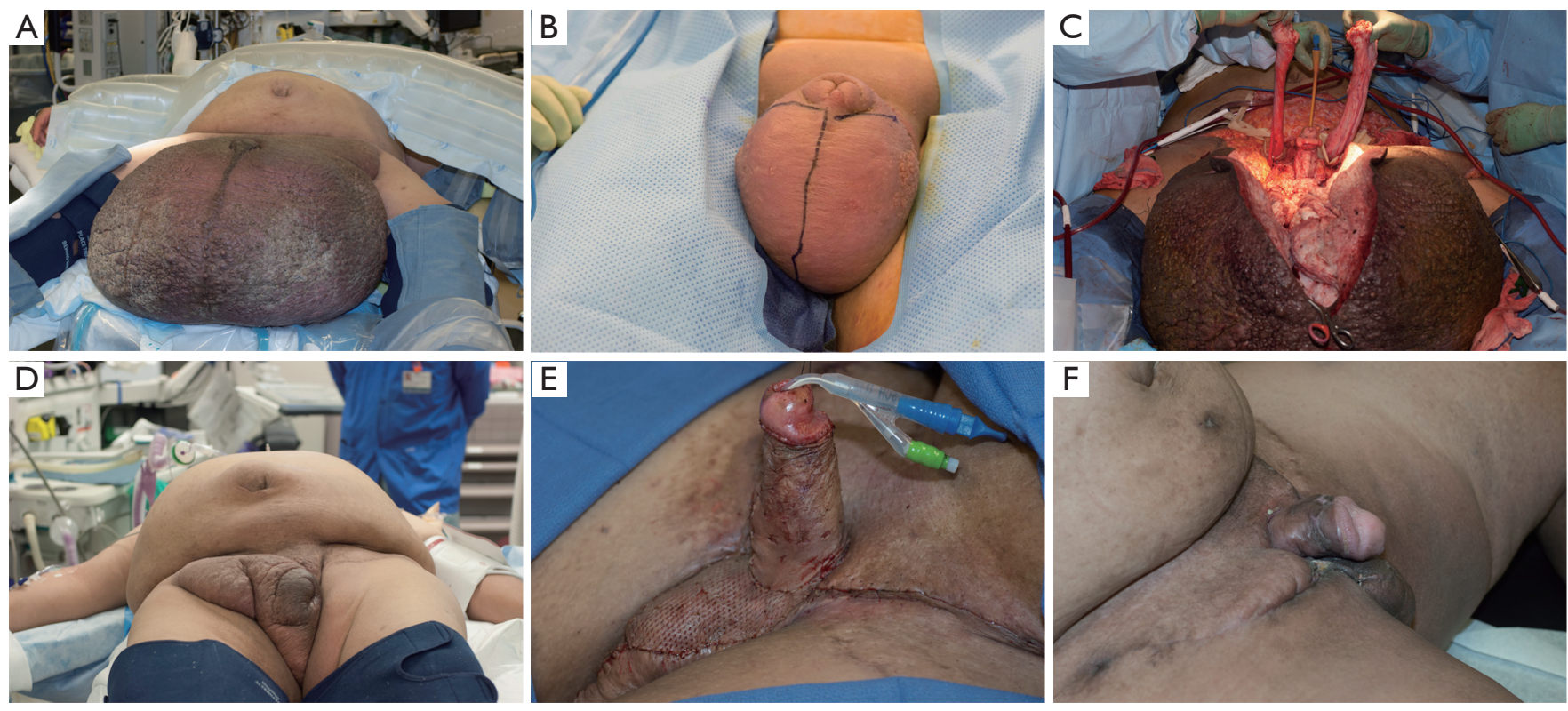

Figure 4 Staged surgical repair of MLL of the scrotum. (A) Preoperative appearance of MLL of the scrotum; (B) T-incision marked out on the scrotum; (C) the spermatic cord and testicles are first isolated prior to removal of any lymphedematous tissue to prevent injury; (D) after the patient has healed from scrotectomy with primary closure, he is brought back into the operating room in a staged fashion to resect residual affected tissue; (E) after all remaining affected tissue is resected, STSG are placed on the penis and scrotum; (F) final cosmetic appearance after skin grafting. MLL, massive localized lymphedema; STSG, split thickness skin grafting.

median raphe (Figure 4B) (17). We first carefully isolate the spermatic cord and testicles to prevent any injury during removal of redundant and lymphedematous scrotal tissue (Figure 4C). Next, we resect all thickened lymphedematous scrotal tissue. While there is often a paucity of viable penile skin tissue, in cases of massive scrotal lymphedema we prefer to initially close the scrotum primarily and cover the penis with local skin flaps. We have found that normal appearing tissue may develop worsening edema after initial resection and that skin grafting in the same setting often leads to poor graft take and healing. Thus, we often stage the procedures and perform skin grafting after the scrotum has full healed and the edematous tissue has been allowed to "declare itself" (Figure 4D-F).

In our series evaluating 11 patients with mean BMI of 60 who had undergone excision of MLL of the scrotum, the masses were successfully removed in every case without testicular injury or loss (17). The surgery was associated with a high rate of local wound complications in 6 patients $(55 \%)$. One patient required reoperation for debridement, but the five remaining patients were successfully managed with local wound care using wet-to-dry packing, which we prefer over wound vac placement due to ease and efficacy.
These patients were noted to have improved quality of life scores, but interestingly, patients gained weight after surgery despite the initial loss of an average of $21 \mathrm{~kg}$ of resection lymphedematous tissue. This is in contrast to findings in a similar series of 12 morbidly obese men with an average BMI of $55 \mathrm{~kg} / \mathrm{m}^{2}$ who underwent buried penis repair who had a similarly high postoperative wound complication rate $(75 \%)$, but observed that $91.7 \%$ of patients lost weight postoperatively in addition to their weight loss during surgical resection of diseased tissue (18). This suggests that MLL associated with morbid obesity is a complex problem and that in additional to a surgical approach, physicians should consider a plan for weight loss and the consideration of bariatric surgery postoperatively if needed (17).

\section{Hidradenitis suppurativa (HS)}

HS is an inflammatory condition of the apocrine glands resulting in recurrent painful, nodular lesions which lead to the development of draining abscesses, fistulas, and sinus tracts. While women are more commonly affected, genital involvement occurs more frequently in men (19). In patients with mild involvement, simple unroofing of 


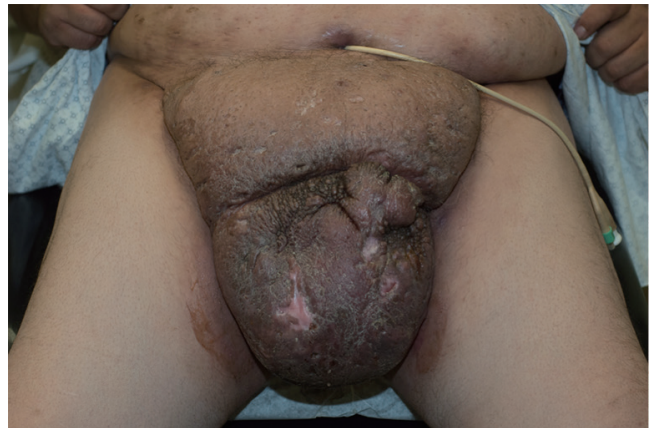

Figure 5 Scrotal hidradenitis suppurativa with involvement of the penis, scrotum, and suprapubic skin.

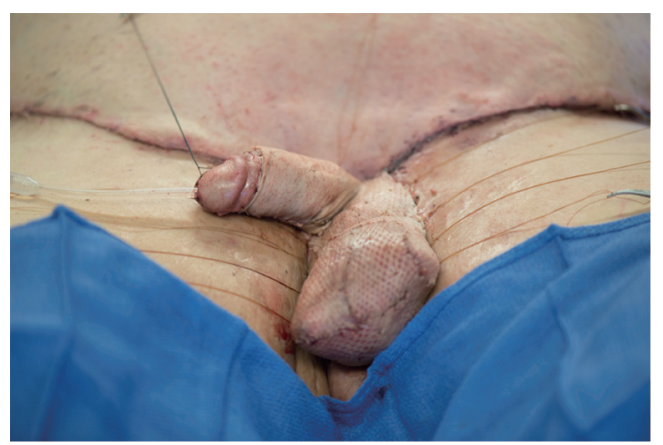

Figure 6 STSG to the scrotum is typically meshed while STSG placed on the penis is unmeshed. STSG, split thickness skin grafting.

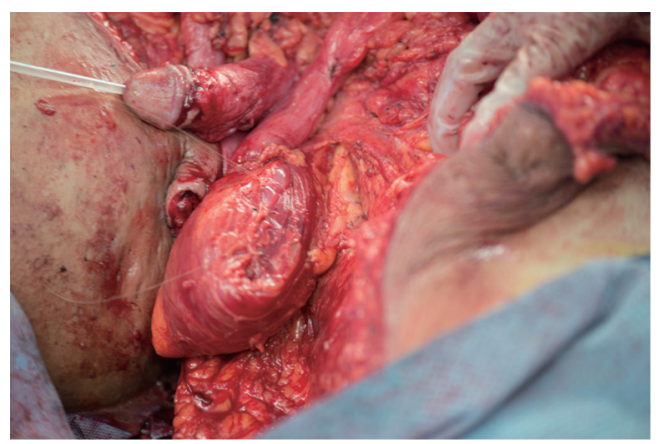

Figure 7 A left gracilis flap encompasses the testicles for added bulk and protection.

abscesses and sinus tracts is adequate. However, in more severe forms chronic skin infection and inflammation leads to skin changes such as scarring, lymphedema, and buried penis (Figure 5). Involvement of the scrotal, penile, and suprapubic skin often necessitate surgical excision with subsequent reconstruction. While these procedures are often associated with complication rates ranging from $17.8 \%$ to $25 \%(20,21)$, a meta-analysis found that relapse rates were $13 \%$ for wide excisions compared to $27 \%$ for deroofing and $22 \%$ for local incisions (22).

In cases of severe genital hidradenitis associated with buried penis, surgical management involves resection of all affected tissue. Preoperatively, we often try to minimize infection and inflammation with a 1-2 weeks course of oral antibiotics. Similar to our approach for buried penis due to MLL, we begin by excising all diseased tissue, taking care to identify and preserve bilateral spermatic cords and testicles, including the tunica vaginalis overlying them. Total scrotectomy is required in patients who have disease extending to the groin creases. Once all disease tissue is resected, attention is turned to coverage of the wound defect. In cases where there is residual normal scrotal skin or medial thigh skin, a local advancement flap can be used to close the defect. We use absorbable sutures in multiple layers in an interrupted fashion given the high rates of wound breakdown and infection. Closed suction drains are placed to prevent seroma formation.

When the patient has insufficient local skin, we prefer to utilize STSG. harvested from the upper lateral thigh using a dermatome set on 0.015 -inch thickness. For scrotal coverage, we mesh the skin 1.5:1 (Figure 6). This has the advantage of decreasing the amount of donor skin needed, decreasing seroma formation, increasing graft take, and the uneven appearance has an acceptable cosmetic result for the scrotum. For penile skin, we use unmeshed STSG and tack it into place with an abundance of 5-0 interrupted chromic sutures. When there is wide excision of the scrotal tissues leaving only the testicles and spermatic cords, skin grafting is compromised by the lack a single smooth rounded surface, and for graft take, the skin graft must be firmly fixed to the recipient bed in all areas of grafting. In these cases, in order to reapproximate the shape of the scrotum and add protective bulk, we sometimes harvest a gracilis flap additional coverage of the testicles and perineum and use the gracilis muscle as the recipient bed for the skin graft (Figure 7). In cases where the involvement extends into the peri-anal area, it may be necessary for a staged approach to be used where the posterior and anterior involved areas are addressed in separate surgeries.

\section{Conclusions}

We have detailed the causes of buried penis, and our 
approach for surgical repair of buried penis in complex cases. Management can be challenging and is largely depends on the etiology of buried penis as well as the degree that local tissues are affected. While these procedures are often associated with a high incidence of wound complications, these are often self-limited and patients experience significant improvement in quality of life measures postoperatively. When combined with postoperative weight loss, surgical repair of buried penis can greatly benefit patients by improving urinary and sexual function in addition to their mental and psychological wellbeing.

\section{Acknowledgements}

None.

\section{Footnote}

Conflicts of Interest: The authors have no conflicts of interest to declare.

Informed Consent: Written informed consent was obtained from the patient for publication of this manuscript and any accompanying images.

\section{References}

1. Rybak J, Larsen S, Yu M, et al. Single center outcomes after reconstructive surgical correction of adult acquired buried penis: a measurement of erectile function, depression, and quality of life. J Sex Med 2014;11:1086-91.

2. Alter GJ. Pubic contouring after massive weight loss in men and women: correction of hidden penis, mons ptosis, and labia major enlargement. Plast Reconstr Surg 2012;130:936-47.

3. Liu F, Lin T, He D, et al. New technique for the treatment of buried penis in children. Urology 2016;88:166-9.

4. Alter GJ, Ehrlich RM. A technique for correct of the hidden penis in children and adults. J Urol 1999;161:455-9.

5. Westerman ME, Tausch TJ, Zhao LC, et al. Ventral slit scrotal flap: a new outpatient surgical option for reconstruction of adult buried penis syndrome. Urology 2015;85:1501-4.

6. Zhao YQ, Zhang J, Yu MS, et al. Functional restoration of penis with partial defect by scrotal skin flap. J Urol 2009;182:2358-61.

7. Folaranmi SE, Corbett HJ, Losty PD. Does application of topical steroids for lichen sclerosis (balanitis xerotica obliterans) affect the rate of circumcision? A systematic review. J Pediatr Surg 2018. [Epub ahead of print].

8. Tausch TJ, Peterson AC. Early aggressive treatment of lichen sclerosis may prevent disease progression. J Urol 2012;187:2101-5.

9. Koifman L, Hampl D, Koifman N, et al. Radical open inguinal lymphadenectomy for penile carcinoma: surgical technique, early complications, and late outcomes. J Urol 2013;190:2086-92.

10. Nelson BA, Cookson MS, Smith JA Jr, et al. Complications of inguinal and pelvic lymphadenectomy for squamous cell carcinoma of the penis: a contemporary series. J Urol 2004;172:494-7.

11. Singh A, Jaipuria J, Goel A, et al. Comparing outcomes of robotic and open inguinal lymph node dissection in patients with carcinoma penis. J Urol 2018. [Epub ahead of print].

12. Lim KH, Speare R, Thomas G, et al. Surgical treatment of genital manifestations of lymphatic filariasis: A systematic review. World J Surg 2015;39:2885-99.

13. Singh V, Sinha RJ, Sankhwar SN, et al. Reconstructive surgery for penoscrotal filarial lymphedema: a decade of experience and follow-up. Urology 2011;77:1228-31.

14. Salako AA, Olanbanji JK, Oladele AO, et al. Surgical reconstruction of giant penoscrotal lymphedema in SubSaharan Africa. Urology 2018;112:181-5.

15. Machol JA 4th, Langenstroer P, Sanger JR. Surgical reduction of scrotal massive localized lymphedema (MLL) in obesity. J Plast Reconstr Aesthet Surg 2014;67:1719-25.

16. Torio-Padron N, Stark GB, Foldi E, et al. Treatment of male genital lymphedema: An integrated concept. J Plast Reconstr Aesthet Surg 2015;68:262-8.

17. Wisenbaugh E, Moskowitz D, Gelman J. Reconstruction of massive localized lymphedema of the scrotum: Results, complications, and quality of life improvement. Urology 2018;112:176-80.

18. Voznesensky MA, Lawrence WT, Keither JN, et al. Patient-reported social, psychological, and urologic outcomes after adult buried penis repair. Urology 2017;103:240-4.

19. Vazquez BG, Alikhan A, Weaver AL, et al. Incidence of hidradenitis suppurativa and associated factors: a population-based study of Olmsted County, Minnesota. J Invest Dermatol 2013;133:97-103.

20. Rompel R, Petres J. Long-term results of wide surgical excision in 106 patients with hidradenitis suppurativa. Dermatol Surg 2000;26:638-43. 
21. Büyükaşik O, Hasdemir AO, Kahramansoy N, et al. Surgical approach to extensive hidradenitis suppurativa. Dermatol Surg 2011;37:835-42.

22. Mehdizadeh A, Hazen PG, Bechara FB, et al. Recurrence

Cite this article as: Ho TS, Gelman J. Evaluation and management of adult acquired buried penis. Transl Androl Urol 2018;7(4):618-627. doi: 10.21037/tau.2018.05.06 of hidradenitis suppurative after surgical management: a systematic review and meta-analysis. J Am Acad Dermatol 2015;73:S70-7. 